\title{
NOTE ON A SYSTEM OF AXIOMS FOR GEOMETRY*
}

BY

\section{ARTHUR RICHARD SCHWEITZER}

$\S 1$.

In these Transactions, vol. 3 (1902), p. 142, E. H. Moore has given a set of projective axioms for geometry. In further development of the standpoint of MOORE a system of axioms for three dimensional Euclidian geometry has been constructed by VEBLEN $\dagger$ by means of the "betweenness" relation. $\ddagger$

A feature of Veblen's system is that a planar axiom (axiom VIII, l. c.) is necessary to establish the existence of an infinitude of points and to prove the theorem, "To any four distinct points of a line the notation $A, B, C, D$ may always be assigned so that they are in the order $A B C D . "$ In this note I give a set of independent axioms in terms of sameness of sense of dyads, abstractly expressed by $\alpha \beta K \gamma \delta \S$, which implies VEBLEN's axioms I-VIII, but which does not require a planar axiom for the proof of the preceding properties. Of the two relations, sameness of sense and betweenness, one is definable in terms of the other; but the two definitions are not equally simple, as will appear below.

\section{$\S 2$.}

The axioms and definitions in terms of the relation $K$ are as follows : $\|$

\section{Axioms.}

1. There exist at least two distinct points.

2. $\alpha \beta \mathrm{K}^{* *}$ implies $\alpha \beta \overline{\mathrm{K}} \beta \alpha .+\dagger$

* Part of a paper presented to the Society (Chicago) April 14, 1906.

†These Transactions, vol. 5 (1904), p. 343.

$\ddagger$ VEBLEN expresses that $B$ is between $A, C$ by saying that $A, B, C$ are in the order $A B C$; thus the triple $A B C$ is an nudefined symbol. In my theory of geometrical relations (which will appear elsewhere in extended form) the order $A B C$ is expressed by the relational statement $(A B C) R(A B C)$; i. e., $A, B, C$ are mutually related.

$\&$ The $\mathrm{K}$ is a symbol of an undefined relation which may be read, "the order $x ;$ is identical with the order $\gamma \delta . "$

II In the phrasing of the axioms I have found the systems of MOORE and VEBLEN instructive. Instead of the relation $\mathrm{K}$ between dyads a class of VAILATI relations between points may be used; I show this in detail elsewhere.

Greek letters are used to denote points.

** That is, $\alpha_{i} 3 \mathrm{~K} \times 3$.

++ The rule over the $K$ is a symbol of negation. 
3. $\alpha \beta \mathrm{K} \gamma \delta$ implies $\beta \alpha \mathrm{KK} \delta \gamma$.

4. $\alpha \neq \beta$ implies the existence of $\xi$ such that either $(\alpha \xi K \alpha \beta$ and $\alpha \xi K \beta \xi)$ or $(\xi \alpha \mathrm{K} \beta \alpha$ and $\xi \alpha \mathrm{K} \xi \beta)$.

5. $\alpha \beta K \gamma \delta$ implies $\gamma \delta \mathrm{K} \alpha \beta$.

5'. $\alpha \beta \mathrm{K} \xi_{\eta}, \xi_{\eta} \mathrm{K} \gamma \delta$ imply $\alpha \beta \mathrm{K} \gamma \delta$.

6. $\alpha \beta \mathrm{K}, \beta \xi \mathrm{K}, \alpha \beta \mathrm{K} \beta \xi$ imply $\alpha \beta \mathrm{K} \alpha \xi$.

6'. $\alpha \beta \mathrm{K} \gamma \delta, \alpha \neq \gamma, \beta \neq \delta$ imply $\alpha \beta \mathrm{K} \alpha \gamma$ or $\alpha \beta \mathrm{K} \gamma \alpha$.

7. $\alpha \beta \mathrm{K}, \beta \gamma \mathrm{K}, \gamma \alpha \mathrm{K}, \alpha \neq \beta, \beta \neq \gamma, \alpha \neq \gamma$ imply the existence of $\xi, \eta, \zeta$ such that $\xi_{\eta} \mathbf{K}, \eta \zeta \mathrm{K}, \zeta \xi \mathrm{K}$ and $\xi \eta \overline{\mathbf{K}} \xi \zeta, \xi \eta \overline{\mathbf{K}} \xi_{\eta}$.

8. $\alpha \beta \mathrm{K}, \beta \gamma \mathrm{K}, \gamma \alpha \mathrm{K}, \alpha \beta \overline{\mathrm{K}} \beta \gamma, \alpha \beta \overline{\mathrm{K}} \gamma \beta, \alpha \beta \mathrm{K} \beta \epsilon, \beta \delta \mathrm{K} \delta \gamma$ imply the existence of $\xi$ such that $\alpha \xi K \xi \gamma$ and $(\delta \epsilon K \xi \epsilon$ or $\delta \epsilon K \delta \xi)$ or $(\epsilon \xi K \delta \xi$ or $\epsilon \xi K \epsilon \delta)$ or $(\xi \delta K \epsilon \delta$ or $\xi \delta K \xi \epsilon)$.*

\section{Definitions.}

1. $\alpha$ is between $\beta \gamma$ means, $\beta \gamma \mathrm{K} \alpha \gamma$ and $\beta \gamma \mathrm{K} \beta \alpha_{0} \dagger$ That is, $\beta \alpha \mathrm{K} \alpha \gamma \mathrm{K} \beta \gamma$, where the order of the dyadic terms, under the axioms, is immaterial.

2. $\alpha$ 1s collinear: with $\beta \gamma$ means, $\beta \gamma \mathrm{K} \alpha \gamma$ or $\beta \gamma \mathrm{K} \beta \alpha$.

Thus, three points $\alpha, \beta, \gamma$ form a triangle if, and only if, $\alpha \beta \mathrm{K}, \beta \gamma \mathrm{K}, \gamma \alpha \mathrm{K}$ and $\beta \gamma \overline{\mathrm{K}} \alpha \gamma, \beta \gamma \overline{\mathrm{K}} \beta \alpha$.

\section{$\S 3$.}

By means of the preceding axioms, the following theorems may be proved. $\neq$

(1) $\alpha \neq \beta$ implies $\alpha \beta \mathrm{K}$.

By 4 we have $\alpha \xi K \alpha \beta$; by $5,5^{\prime}, \alpha \beta \mathrm{K}$.

(2) $\alpha \beta \overline{\mathbf{K}} \gamma \delta$ implies $\alpha \beta \overline{\mathbf{K}} \delta \gamma, \beta x \overline{\mathbf{K}} \gamma \delta$.

By $5,5^{\prime}, \alpha \beta \mathrm{K} \gamma \delta$ implies $\alpha \beta \mathrm{K}, \gamma \delta \mathrm{K}$ and hence by $2, \alpha \beta \overline{\mathbf{K}} \beta \alpha, \gamma \delta \overline{\mathrm{K}} \delta \gamma$. Now if $\alpha \beta \mathrm{K} \delta \gamma$, since $\alpha \beta K \gamma \delta$ we have by 5 and $5^{\prime} \gamma \delta \mathrm{K} \delta \gamma$, which contradicts axiom 2. Similarly we prove $\beta \alpha \overline{\mathbf{K}} \gamma \delta$.

(3) $\alpha \beta$ K $\gamma \delta$ implies $\alpha \neq \beta$ and $\gamma \neq \delta$.

By theorem (2).

(4) If $\alpha$ is between $\beta \gamma$ then $\alpha$ is between $\gamma \beta$ and $\beta$ is not between $\alpha \gamma$; also $\alpha, \beta, \gamma$ are distinct.

By definition 1 and the hypothesis we have $\beta \gamma K \alpha \gamma$ and $\beta \gamma K \beta \alpha$; hence by 3 , $\gamma \beta \mathrm{K} \gamma \alpha$ and $\gamma \beta \mathrm{K} \alpha \beta$.

If $\beta$ is between $\alpha \gamma$ we have $\alpha \gamma \mathrm{K} \alpha \beta$ and $\alpha \gamma \mathrm{K} \beta \gamma$; hence by $5,5^{\prime} \beta \gamma \mathrm{K} \alpha \beta$, which contradicts $\beta \gamma \mathrm{K} \beta x$ by (2).

From $\gamma \beta \mathrm{K}_{\gamma x}$ and $\gamma \beta \mathrm{K} \alpha \beta$, by (3) it follows that $\alpha, \beta, \gamma$ are distinct.

(5) If $\alpha \neq \beta$ then there is a point $\xi^{\prime}$ such that $\beta$ is between $\alpha \xi^{\prime}$, and a point $\xi^{\prime \prime}$ such that $\alpha$ is between $\beta \xi^{\prime \prime}$.

* Under the axioms the terms of the major disjunctions are equivalent.

† Compare with this definition Grassyann, Gesammelte Werke, vol. I, p. 7.

$\ddagger$ In the demonstrations. numbers without parentheses refer to axioms of $\$ 2$; numbers enclused in parentheses refer to theorems. 
By 4 , if $\alpha \neq \beta$ there exists $\xi^{\prime}$ such that $\beta$ is between $\alpha \xi^{\prime}$; interchanging $x$ and $\beta$, we find by 4 a point $\xi^{\prime \prime}$ such that $\alpha$ is between $\beta \xi^{\prime \prime}$.

$\left(6^{\prime}\right) \alpha \beta \mathrm{K} \gamma \beta, \gamma \neq \alpha$ imply $\alpha \beta \mathrm{K} \gamma \alpha$ or $\alpha \beta \mathrm{K} \alpha \gamma$.

Since by $(3) \gamma \neq \beta$, there exists by $4,5,5^{\prime}$ a point $\xi$ such that $\gamma \beta K \beta \xi$, i. e., by $6, \gamma \beta \mathrm{K}_{\gamma} \xi$. From $\alpha \beta \mathrm{K}_{\gamma} \beta$ and $\gamma \beta \mathrm{K}_{\gamma} \xi$ it follows by $5^{\prime}$ that $\alpha \beta \mathrm{K}_{\gamma} \xi$. Hence by $6^{\prime}$, since $\gamma \neq \alpha$ and $\xi \neq \beta, \alpha \beta \mathrm{K} \alpha \gamma$ or $\alpha \beta \mathrm{K} \gamma \alpha$.

(6) If $\xi$ is collinear with $\beta \gamma$ and $\xi \neq \beta, \gamma$, then $\xi$ is between $\beta \gamma$ or $\beta$ is between $\xi \gamma$ or $\gamma$ is between $\xi \beta$, and conversely.

By definition and the hypothesis, $\beta \gamma \mathrm{K} \xi \gamma$ or $\beta \gamma \mathrm{K} \beta \xi$. If $\beta \gamma \mathrm{K} \xi \gamma$, by $\left(6^{\prime}\right)$ we have $\beta \gamma \mathrm{K} \beta \xi$ or $\beta_{\gamma} \mathrm{K} \xi \beta$. If $\beta_{\gamma} \mathrm{K} \xi \beta$, by $5,5^{\prime}, 6, \beta_{\gamma} \mathrm{K} \xi \beta \mathrm{K} \xi \gamma$ and hence $\beta$ is between $\xi \gamma$. If $\beta \gamma \mathrm{K} \beta \xi$, by $\left(6^{\prime}\right), 3, \beta \gamma \mathrm{K} \xi \gamma$ or $\beta \gamma \mathrm{K} \gamma \xi$. If $\beta \gamma \mathrm{K} \beta \xi$ and $\beta \gamma \mathrm{K} \xi \gamma$ then $\xi$ is between $\beta_{\gamma}$; if $\beta \gamma \mathrm{K} \beta \xi$ and $\beta_{\gamma} \mathrm{K} \gamma \xi$ then $\gamma$ is between $\xi \beta$.

Conversely, if $\xi$ is between $\beta \gamma$ then $\beta \gamma \mathrm{K} \beta \xi$; if $\beta$ is between $\xi \gamma, \beta \gamma \mathrm{K} \xi \gamma$; if $\gamma$ is between $\xi \beta, \beta \gamma \mathrm{K} \beta \xi$.

(7) If $\gamma \neq \delta$ are collinear with $\alpha \beta$ then $\alpha$ is collinear with $\gamma \delta$.

By the hypothesis and definition 2, $\alpha \beta \mathrm{K} \alpha \gamma$ or $\alpha \beta \mathrm{K}_{\gamma} \beta$ and $\alpha \beta \mathrm{K} \alpha \delta$ or $\alpha \beta \mathrm{K} \delta \beta$. To prove, $\gamma \delta \mathrm{K} \gamma \alpha$ or $\gamma \delta \mathrm{K} \alpha \delta$. We distinguish four cases, labelled I, II, III, IV respectively.

I. $\alpha \beta \mathrm{K} \alpha \gamma, \alpha \beta \mathrm{K} \delta \beta$.

By $\left(6^{\prime}\right) \alpha \beta \mathrm{K} \delta \beta$ implies $\alpha \beta \mathrm{K} \delta x$ or $\alpha \beta \mathrm{K} \alpha \delta . \quad \alpha \beta \mathrm{K} \delta \alpha$ and $\alpha \beta \mathrm{K} \alpha \gamma$ imply by $5,5^{\prime}$, $6,3, \gamma \delta \mathrm{K} \alpha \delta$. $\alpha \beta \mathrm{K} \alpha \delta$ and $\alpha \beta \mathrm{K} \alpha \gamma$ imply by $5,5^{\prime}, \alpha \delta \mathrm{K} \alpha \gamma$. By $\left(6^{\prime}\right) \alpha \delta \mathrm{K} \alpha \gamma$ implies $\alpha \delta \mathrm{K} \gamma \delta$ or $\alpha \delta \mathrm{K} \delta \gamma$; if $\alpha \delta \mathrm{K} \delta \gamma$, by $6, \alpha \delta \mathrm{K} \alpha \gamma$ and hence $\gamma \delta \mathrm{K} \gamma \alpha$.

II. $\alpha \beta \mathrm{K} \alpha \gamma, \alpha \beta \mathrm{K} \alpha \delta$.

Hence $\alpha \gamma \mathrm{K} \alpha \delta$; therefore by (6') $\alpha \gamma \mathrm{K} \gamma \delta$ or $\alpha \gamma \mathrm{K} \delta \gamma$. If $\alpha \gamma \mathrm{K} \gamma \delta$, then $\gamma \delta \mathrm{K} \alpha \delta$. If $\alpha \gamma \mathrm{K} \delta \gamma$ then $\gamma \delta \mathrm{K} \gamma \alpha$.

III. $\alpha \beta \mathrm{K} \gamma \beta, \alpha \beta \mathbf{K} \alpha \delta$.

$\alpha \beta \mathrm{K} \gamma \beta$ implies $\alpha \beta \mathrm{K} \gamma \alpha$ or $\alpha \beta \mathrm{K} \alpha \gamma$. If $\alpha \beta \mathrm{K} \alpha \gamma$ and $\alpha \beta \mathrm{K} \alpha \delta$ then we have II. If $\alpha \beta K \alpha \delta$ and $\alpha \beta \mathrm{K} \gamma \alpha$ then $\gamma \alpha \mathrm{K} \alpha \delta$, that is, $\gamma \delta \mathrm{K} \gamma \alpha$.

IV. $\alpha \beta \mathrm{K} \gamma \beta, \alpha \beta \mathrm{K} \delta \beta$.

$\alpha \beta \mathrm{K} \gamma \beta$ implies $\alpha \beta \mathrm{K} \gamma \alpha$ or $\alpha \beta \mathrm{K} \alpha \gamma$. For $\alpha \beta \mathrm{K} \alpha \gamma$ and $\alpha \beta \mathrm{K} \delta \beta$ see I. If $\alpha \beta \mathrm{K}_{\gamma x}$ then $\gamma \alpha \mathrm{K} \gamma \beta, \alpha \beta \mathrm{K} \gamma \beta$. Also $\alpha \beta \mathrm{K} \gamma \beta, \alpha \beta \mathrm{K} \delta \beta$ imply $\gamma \beta \mathrm{K} \delta \beta$; hence $\gamma \beta \mathrm{K} \gamma \delta$ or $\gamma \beta \mathrm{K} \delta \gamma$. If $\gamma \beta \mathrm{K} \gamma \delta$, since $\gamma \alpha \mathrm{K} \gamma \beta, \gamma \alpha \mathrm{K} \gamma \delta$ and hence $\gamma \delta \mathrm{K} \gamma \alpha$. Sim. ilarly, if $\gamma \beta \mathrm{K} \delta \gamma$ then $\gamma \delta \mathrm{K} \alpha \delta$.

(8) There exists an infinitude of points.

By axiom 1 and the repeated application of 4 , using $5,5^{\prime}$ we find points $\xi_{1}, \xi_{2}, \cdots, \xi_{n}$ such that

$$
\alpha \beta \mathrm{K} \beta \xi_{1} \mathrm{~K} \xi_{1} \xi_{2} \mathrm{~K} \ldots \mathrm{K} \xi_{n-1} \xi_{n} .
$$

By axioms 6 and 2 the points $\xi_{1}, \xi_{2}, \ldots, \xi_{n}$ are distinct. This proof, of course involves the principle of induction.

The proof of the order theorem mentioned in $\S 1$ is easily obtained by prov- 
ing VebLeN's six lemmas, l. c., pp. 356-357 with the aid of 1-6' only ;* a direct proof is also readily given.

$$
\S 4 \text {. }
$$

The independence systems for axioms 1-8 are very simple. We denote by $S_{i}$ the set of elements such that in this set axiom $i(i=1,2,3, \ldots, 8)$ is contradicted and the remaining axioms are satisfied or non-effective. $\dagger$

$$
S_{1}
$$

One point, 1 , such that $11 \overline{\mathrm{K}}$. Axiom 1 is contradicted; the remaining axioms are non-effective.

$$
S_{2} \text {. }
$$

Two points 1, 2, such that any two dyads (identical or not) of the following set are in the $K$ relation: 11, 22, 12, 21. Axiom 1 is satisfied; axiom 2 is contradicted; axiom 3 is satisfied. Axiom 4 asserts $1 \neq 2$ implies the existence of $\xi$ such that $1 \xi \mathrm{K} 12$ and $1 \xi \mathrm{K} 2 \xi$ or $\xi 1 \mathrm{~K} 21$ and $\xi 1 \mathrm{~K} \xi 2$; thus we may put $\xi=1$ or 2 and hence axiom 4 is satisfied. Axioms $5,5^{\prime}, 6,6^{\prime}$ are easily verified. Axioms 7 and 8 are non-effective.

$$
S_{3} \text {. }
$$

A Vallati line : $\ddagger \alpha \beta \mathrm{K} \gamma \delta$ if, and only if, $\alpha$ precedes $\beta$ and $\gamma$ precedes $\delta$; $\alpha \beta \mathrm{K}$ if, and only if, $\alpha$ precedes $\beta$. Axioms 1,2 are satisfied; axiom 3 is contradicted. Axiom 4 is satisfied: the terms of the disjunction are mutually exclusive. Axioms 5, 5',6, 6' are satisfied. Axioms 7,8 are non-effective, since $\alpha \beta \mathrm{K}$, $\beta_{\gamma} \mathrm{K}$ imply $\alpha \gamma \mathrm{K}$.

$$
S_{4} \text {. }
$$

Two points, 1,2 , such that $12 \mathrm{~K}, 21 \mathrm{~K}, 12 \overline{\mathrm{K}} 21,21 \overline{\mathrm{K}} 12$. Axioms $1-3$ are satisfied. Axiom 4 is contradicted. Axioms 5, $5^{\prime}$ are satisfied. Axiom 6 is non-effective, since $12 \overline{\mathrm{K}} 21,21 \overline{\mathrm{K}} 12$. Axiom $6^{\prime}$ is non-effective since only $12 \mathrm{~K}$, $21 \mathrm{~K}$. Since there are only two points axioms 7,8 are non-effective.

$$
S_{5} \text {. }
$$

Two points, 1,2 , such that $11 \mathrm{~K} 12,11 \mathrm{~K} 21,12 \overline{\mathrm{K}} 11,21 \overline{\mathrm{K}} 11,22 \mathrm{~K} 21,22 \mathrm{~K} 12$, $21 \overline{\mathrm{K}} 22,12 \overline{\mathrm{K}} 22,11 \overline{\mathrm{K}}, 12 \overline{\mathrm{K}}, 22 \overline{\mathrm{K}}, 21 \overline{\mathrm{K}}, 11 \overline{\mathrm{K}} 22,22 \overline{\mathrm{K}} 11$. Axiom 1 is satisfied. Axiom 2 is non-effective. Axiom 3 is satisfied. Axiom 4 is satisfied since $1 \neq 2$ implies 1,2 such that $11 \mathrm{~K} 12$ and $11 \mathrm{~K} 21$ and $22 \mathrm{~K} 21$ and $22 \mathrm{~K} 12$. Axiom 5 is contradicted. Axioms $5{ }^{\prime}-8$ are non-effective.

$$
S_{5^{\prime}} \text {. }
$$

Two points 1,2 such that $11 \mathrm{~K} 12,12 \mathrm{~K} 11,22 \mathrm{~K} 21,21 \mathrm{~K} 22,11 \mathrm{~K} 21,21 \mathrm{~K} 11$, $22 \mathrm{~K} 12,12 \mathrm{~K} 22,11 \overline{\mathrm{K}}, 12 \overline{\mathrm{K}}, 22 \overline{\mathrm{K}}, 21 \overline{\mathrm{K}}, 11 \overline{\mathrm{K}} 22,22 \overline{\mathrm{K}} 11$. Axiom 1 is satisfied.

* As in the case of the preceding theorems, axiom $\sigma^{\prime}$ is user here only through theorem $\left(6^{\prime}\right)$.

† The notation $S_{i}$ is due to Drckson, these Transactions, vol. 4 (1903), p. 16.

$\ddagger$ Rivista di Matematica II, pp. 71-75. 
Axiom 2 is non-effective. Axiom 3 is satisfied. Axiom 4 requires that $1 \neq 2$ implies the existence of $\xi$ such that $1 \xi \mathrm{K} 12$ and $1 \xi \mathrm{K} 2 \xi$ or $\xi 1 \mathrm{~K} 21$ and $\xi 1 \mathrm{~K} \xi 2$; hence we may take $\xi=1$. Similarly, we must have $\xi$ such that $2 \xi K 21$ and $2 \xi \mathrm{K} 1 \xi$ or $\xi 2 \mathrm{~K} 12$ and $\xi 2 \mathrm{~K} \xi 1$; hence we may take $\xi=2$. Axiom 5 is satisfied, axiom $5^{\prime}$ is contradicted. Axioms 6-8 are non-effective.

$$
S_{6} \text {. }
$$

Seven points, 1-7, arranged in the following two sets of 21 dyads each:

\begin{tabular}{c|c} 
I & II \\
$12,23,34,45,56,67,71$ & $21,32,43,54,65,76,17$ \\
$13,24,35,46,57,61,72$ & $31,42,53,64,75,16,27$ \\
$41,52,63,74,15,26,37$ & $14,25,36,47,51,62,73$
\end{tabular}

$\alpha \beta \mathrm{K} \gamma \delta$ if, and only if, $\alpha \beta$ and $\gamma \delta$ are both of set I or both of set II. $\alpha \beta \mathrm{K}$ if, and only if, $\alpha \beta$ is of set I or of set II. Axioms 1-5' are satisfied. Axiom 6 is contradicted. Axioms $6^{\prime}-7$ are satisfied; axiom 8 is non-effective.

$$
S_{6^{\prime}} \text {. }
$$

A cartesian plane having rational coördinates ; $\alpha \beta \mathrm{K} \gamma \delta$ if, and only if, $\alpha \beta$ and $\gamma \delta$ are two parallel segments similarly directed. Axiom $6^{\prime}$ is contradicted. In verifying the remaining axioms we note that two parallel segments are collinear if they pass through the same point, and that any segment is parallel to itself. We observe that the property expressed by theorem $\left(6^{\prime}\right)$ under $\S 3$ is verified.*

$$
S_{7} \text {. }
$$

A cartesian line having integral coördinates; $\alpha \beta \mathrm{K} \gamma \delta$ if, and only if, the segment $\alpha \beta$ has the same sign as the segment $\gamma \delta$.

$$
S_{8} \text {. }
$$

A cartesian plane having integral coördinates.

\section{$\S 5$.}

To define $\alpha \beta K \gamma \delta$ in terms of VeBLEN's order, we find it desirable to introduce a point. We define as follows :

$\alpha \beta \mathrm{K} \gamma \delta$ means, $1^{\circ}$. There exists a $\xi$ such that $(\alpha \beta \xi$ and $\gamma \delta \xi)$ or $(\beta \alpha \xi$ and $\delta \gamma \xi)$. $2^{\circ}$. The existence of $\xi$ such that $\alpha \beta \xi$ and $\gamma \delta \xi$ implies $\alpha \gamma \xi$ or $\gamma \beta \xi$; and the existence of $\xi$ such that $\beta \alpha \xi$ and $\delta \gamma \xi$ implies $\beta \delta \xi$ or $\delta \alpha \xi$.

* This remark is important if we wish to extend axioms 1-8 to a set of axioms for Euclidian geometry (cl. VeBLEN, loc. cit.). Such extension may easily be made eitber by retaining axiom $6^{\prime}$ as stated, or by substituting for the latter an axiom with the content of theorem $\left(6^{\prime}\right)$. In each instance the parallel axiom will have a corresponding phrasing, and the relation $K$ may be interpreted respectively by sameness of sense of (collinear) segments, similarity of direction of (parallel) segments. 
In virtue of this definition it might seem that we could establish a correspondence between "sameness of sense" properties and "order" properties. This view seems, however, largely unprofitable; for example, that three points are collinear can be expressed much more concisely in terms of order than is inferred by this correspondence from the definition in terms of the relation K.† Thus it does not seem possible to convert definitionally one system of axioms into the other and to retain that elegance which is peculiar to each individual standpoint.

† VerLen, l. c. 